\title{
Charge Transport Characterization of PbS Quantum Dot Solids for High Efficiency Solar Cells
}

\author{
Young Jin Jeong ${ }^{1,3}$, Jihoon Jang ${ }^{1}$, Jung Hoon Song ${ }^{1,4}$, Hyekyoung Choi ${ }^{1,2}$, \\ Sohee Jeong ${ }^{1,2 \dagger}$, and Seung Jae Baik ${ }^{3}$ \\ ${ }^{l}$ Nanomechanical Systems Research Division, Korea Institute of Machinery and Materials, \\ Daejeon 305-343, Korea \\ ${ }^{2}$ Department of Nano Mechatronics, Korea University of Science and Technology, Daejeon 305-343, Korea \\ ${ }^{3}$ Department of Electrical, Electronic, and Control Engineering, Hankyong National University, \\ Anseong 456-749, Korea \\ ${ }^{4}$ Graduate School of Nanoscience and Technology, KAIST, Daejeon 305-701, Korea
}

(Received January 5, 2015 : revised February 24, 2015 : accepted February 26, 2015)

\begin{abstract}
The $\mathrm{PbS}$ quantum dot is an emerging photovoltaic material, which may provide high efficiency breakthroughs. The most crucial element for the high efficiency solar cells's development is to understand charge transport characteristics of $\mathrm{PbS}$ quantum dot solids, which are also important in planning strategic research. We have investigated charge transport characteristics of $\mathrm{PbS}$ quantum dot solids thin films using space charge limited conduction analysis and assessed thickness dependent photovoltaic performances. The extracted carrier drift mobility was low $-10^{-2} \mathrm{~cm}^{2} / \mathrm{Vs}$ with the estimated diffusion length about $50 \mathrm{~nm}$. These and recently reported values were compared with those from a commercial photovoltaic material, and we present an essential element in further development of $\mathrm{PbS}$ quantum dot solids materials.
\end{abstract}

\footnotetext{
Keywords : Colloidal quantum dot, Quantum dot solids, Space charge limited conduction, Thickness dependence of photovoltaic performance

OCIS codes : (160.5335) Photosensitive materials; (160.6000) Semiconductor materials; (310.6845) Thin film devices and applications; (310.3840) Materials and process characterization
}

\section{INTRODUCTION}

Colloidal quantum dot (CQD) based solar cells have recently gotten much attention with a rapid growth in their efficiency development [1-4]. Initially multi-exciton generation phenomena in QD materials have driven CQD photovoltaics [5-8], which are now becoming one of the competitors for the next generation thin film solar cells with a lower cost per watt. Among the technology breakthroughs in CQD photovoltaics, the most fundamental and crucial achievement is in the CQD surface passivation in forming quantum dot solids (QDSs) [9-12]. The fundamental limitation of charge transport in QDS is summarized by QD to QD tunneling resistance and QD surface states charge trapping. These have been alleviated by applying passivation ligands with short molecular chain lengths like mercaptopropionic acid (MPA) or ethanedithiol (EDT). Additional innovation in QD surface passivation has been achieved by applying atomic halide passivation coupled with the above ligand materials [9]. As a result, the best reported efficiency until this time is above $8.5 \%$ [2].

The electrical characterization of QDS film quality has been performed by quantifying carrier drift mobilities, recombination lifetimes, and their coupled measures such as diffusion or drift lengths. Indeed, those coupled measures are more direct indications of the film photovoltaic quality, whose signature is directly observed in thickness dependent photovoltaic performances [1]. Once we know the quantitative characterization results, the competitiveness of the QDS materials and solar cells is clearly revealed by comparing those values with other solar cell technologies. This is a crucial aspect in establishing research and development scenarios in this emerging field. In this work, we have characterized electrical transport in $\mathrm{PbS}$ QDS using space charge limited conduction (SCLC) and thickness dependent photovoltaic performances [13].

\section{※Corresponding authors: *sjbaik@hknu.ac.kr, 'sjeong@kimm.re.kr}

Color versions of one or more of the figures in this paper are available online. 
From these data with other literature values [1], we would like to assess state-of-the-art technology of CQD photovoltaics in comparison with existing commercial photovoltaics.

\section{EXPERIMENTAL DETAILS}

We prepared PbS CQD as described previously [14], a typical absorption spectrum of which is shown in Fig. 1.

\subsection{Device Fabrication for SCLC Characterization}

Within a $\mathrm{N}_{2}$-filled glove box PbS QD solar cells were fabricated using a layer-by-layer spin-coating technique onto ITO substrates, which were pre-cleaned by sonication for 10 minutes sequentially in acetone, methanol, and de-ionized (DI) water. PbS QDS (10 mg ml ${ }^{-1}$ in octane) was coated on the substrate by a spin coating method with $2500 \mathrm{rpm}$, which was treated by MPA ( 1 vol. \% in methanol) and subsequently rinsed by methanol. This coating cycle was repeated around 10 times until the thickness of the overall PbS QDS layer became $100 \mathrm{~nm}$, which was estimated by cross-sectional FE-SEM. The films were further annealed at $90{ }^{\circ} \mathrm{C}$ for 5 $\mathrm{min}$ in air. Finally, a thin LiF layer of $1 \mathrm{~nm}$ and $\mathrm{Al}$ back electrode of $200 \mathrm{~nm}$ were sequentially deposited on the $\mathrm{PbS}$ QDS layer using a thermal evaporator.

\subsection{Heterojunction PbS QD Solar Cells Fabrication}

$\mathrm{TiO}_{2}$ layers were prepared by spin coating $\mathrm{TiO}_{2}$ paste (Solarronix, Ti-Nanoxide, HT/SC) on fluorine-doped tin oxide (FTO) substrate at 3,000 rpm and annealing at $450{ }^{\circ} \mathrm{C}$ for $30 \mathrm{~min}$. PbS QDs layers were then fabricated using layerby-layer spin-coating methods using PbS QDs $\left(60 \mathrm{mg} \mathrm{ml}^{-1}\right.$ in octane) and MPA ( 1 vol.\% in methanol) as described previously [14]. The films were further annealed at $90{ }^{\circ} \mathrm{C}$ for $5 \mathrm{~min}$ in air. Finally, a $\mathrm{MoO}_{3}$ layer of about $10 \mathrm{~nm}$ and $\mathrm{Au} / \mathrm{Ag}$ back electrode of $50 / 150 \mathrm{~nm}$ were sequentially deposited using a thermal evaporator. Six solar cells were

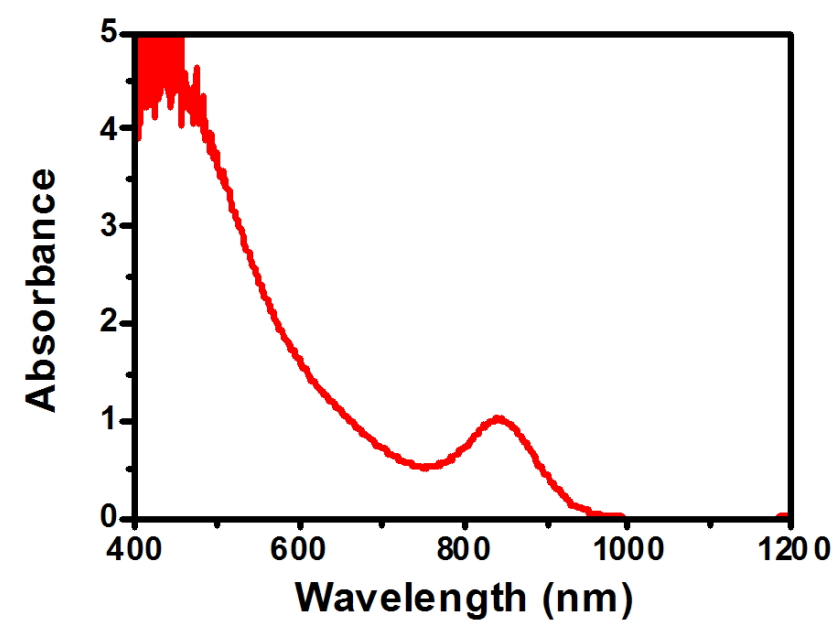

FIG. 1. Light absorption spectrum of PbS CQDs dispersed in TCE solution. fabricated in one batch experiment to consider characteristic variation.

Absorption spectra of PbS QDs in trichloroethylene (TCE) solutions and solar cells were measured by ultraviolet-visible (UV-VIS) spectroscopy (Shimadzu, UV3600) using an integrating sphere. Current-voltage (I-V) curves were measured using a Keithley 2400 source meter. A solar simulator (94023A, New port INC) with a 450W Xe lamp light source operating at $100 \mathrm{~mW} \mathrm{~cm}{ }^{-2}$ as calibrated by a standard silicon solar cell was used to simulate the solar spectrum under AM1.5G conditions. External quantum efficiency (EQE) measurements were conducted on a custom built set-up (SPIQE200-5327, New port INC) which consists of 300W Xe lamp, a power supply, a monochrometer generating $20 \mathrm{~nm}$ band width, and a Merlin lock-in amplifier. A beam size of $0.01 \mathrm{~cm}^{2}$ was used to excite solar devices with active area of 0.09 $\mathrm{cm}^{2}$. All current-voltage and EQE measurements were carried out in an $\mathrm{N}_{2}$ environment.

\section{RESULTS AND DISCUSSION}

Space charge limited conduction (SCLC) governs charge transport in very thin QDS films, where QDS is thin enough to provide formation of percolation paths [13]. That is, the device for the characterization of SCLC needs to be nonrectifying, and therefore, the heterojunction solar cell structure cannot be used for the SCLC characterization. This behaviour gives a useful tool for characterizing charge carrier mobility, carrier concentration, and trap density related to the transport in a direct way from simple I-V measurements. We have fabricated ITO/PbS QDS/Al structure with $100 \mathrm{~nm}$ thick $\mathrm{PbS}$ QDS, and measured I-V characteristics to extract abovementioned parameters according to the model described before [13]. The parameter extraction was based on the single trap SCLC model, where hole unipolar drift transport was assumed. We have found hysteretic I-V behaviour as shown in Fig. 2(a), which is physically related to the efficiency measurement problems recently issued for assessing emerging solar cells based on quantum dots [12] or perovskite materials [15]. The physical mechanism may be related to free carrier trapping or bound charge polarization, and in any case, it would be reasonable to assume a change in the electrostatic energy barrier to explain the hysteresis. The voltage shift in the hysteresis is found to be around $0.2 \mathrm{~V}$, which corresponds to $0.7 \times 10^{17} \mathrm{~cm}^{-3}$ volume charges if we assume uniform volume charges (in this calculation, the dielectric constant of the PbS QDS was 15 [13]). This may be related to the volume trap density, if most of the traps are filled with free carriers; however, this is smaller than the value obtained from SCLC analysis implying partially filled trap sites in hysteresis sweep. The mobility values show the order of $10^{-2} \mathrm{~cm}^{2} / \mathrm{Vs}$ as shown in Fig. 2(b), where they exhibit a slightly smaller value in the reverse sweep. Smaller extracted mobility in the reverse sweep may be due to the electrostatic scattering due to trapped charges. The carrier concentration shown in Fig. 2(c) 


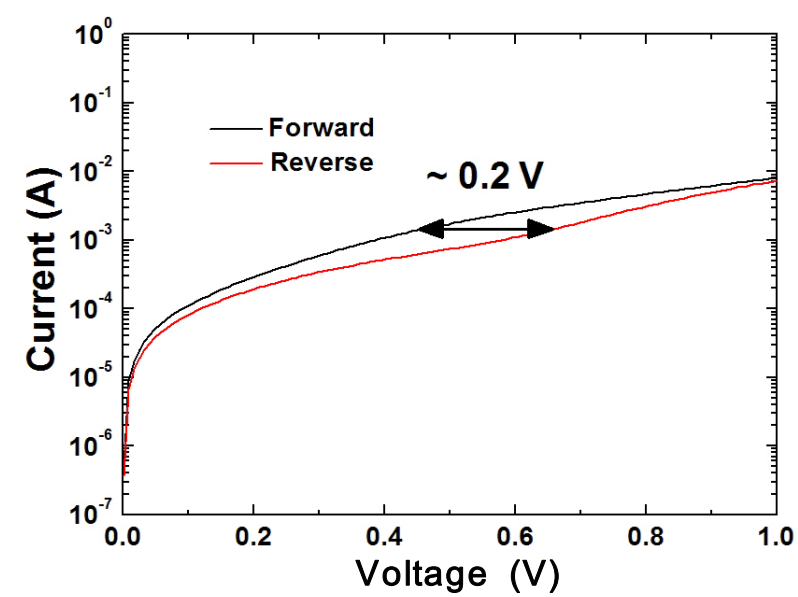

(a)

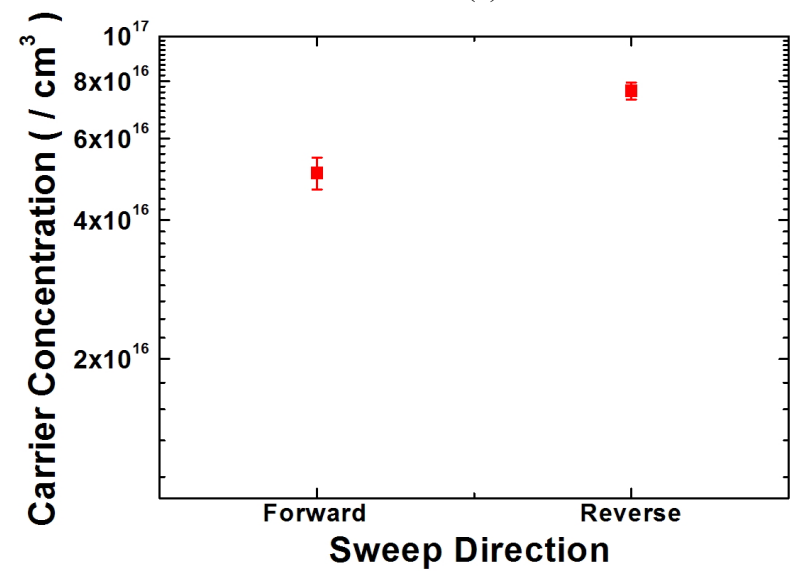

(c)

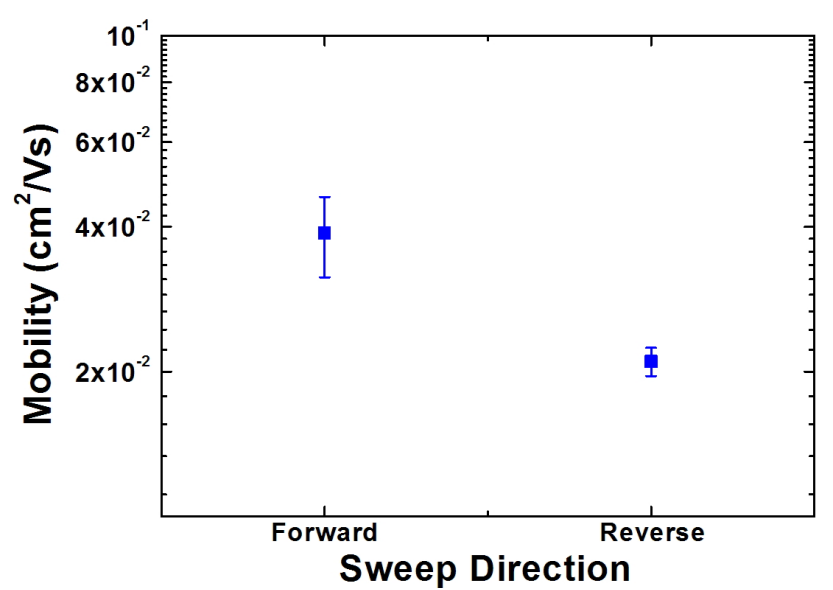

(b)

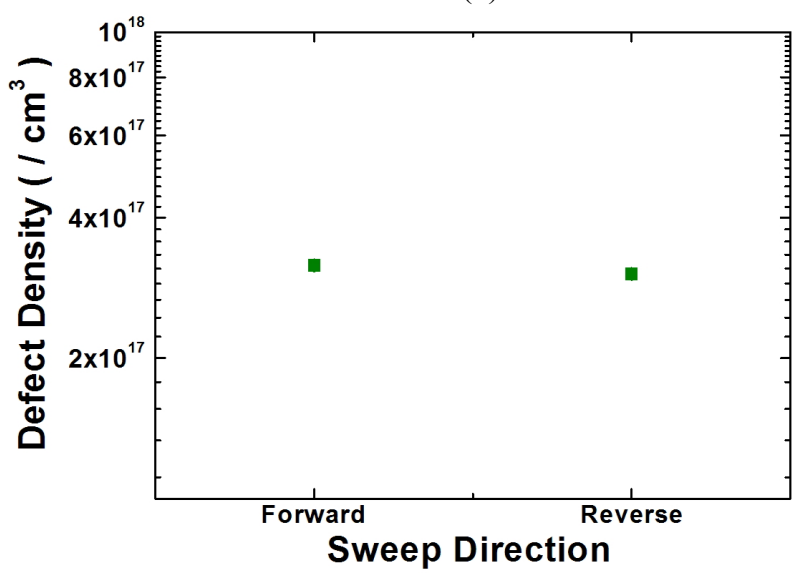

(d)

FIG. 2. (a) Typical current vs. voltage characteristics of ITO/PbS QDS/LiF/Al device with hysteretic behavior. Mobility (b), carrier concentration (c), and defect density values (d), extracted by SCLC analyses. Data from five different devices are used.

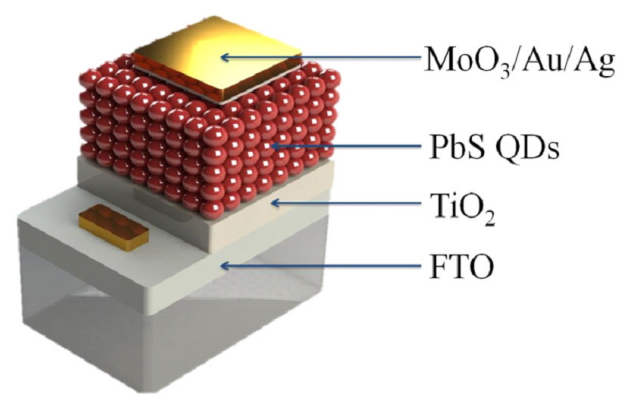

(a)

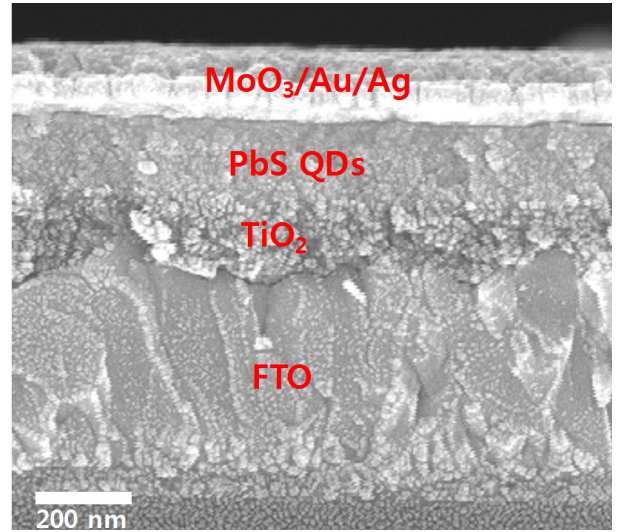

(b)

FIG. 3. (a) Device scheme for thickness dependent characterization, and (b) cross sectional SEM image for the $200 \mathrm{~nm}$-thick solar cell.

exhibits an opposite trend, where a slightly larger extracted carrier concentration from the reverse sweep may be due to additional participation of detrapped charges in the current flow. The extracted trap density from SCLC analysis in Fig. 2(d) is around $3 \times 10^{17} \mathrm{~cm}^{-3}$, which may be a more reasonable value than that from the $\mathrm{I}-\mathrm{V}$ hysteresis shown above.

Solar cells with various PbS QDS thicknesses were fabricated as shown in Fig. 3 (a) and (b), which give a series of photovoltaic performances as shown in Fig. 4. The increase of 
the thickness monotonically reduces fill factor $(\mathrm{FF})$, while open circuit voltage $\left(\mathrm{V}_{\mathrm{oc}}\right)$ and short circuit current density $\left(\mathrm{J}_{\mathrm{sc}}\right)$ increase up to a critical thickness and decrease again with a thickness thicker than the critical thickness. Similar thickness dependent behaviours have been reported before in $\mathrm{PbS}$ based quantum dot solar cells $[1,13]$ before, and the critical thickness that determines the $\mathrm{J}_{\mathrm{sc}}$ is thought to be the onset thickness of full depletion. From Fig. 4(b) and (c), the onset thickness of full depletion is around 300 $\mathrm{nm}$, above which, the dominant carrier collection mechanism switches from drift to diffusion.

The critical thickness that represents the thickness dependence of conversion efficiency has a functional relationship with the carrier diffusion length [1]. The critical thickness around $250 \mathrm{~nm}$ in this experiment roughly corresponds to the characteristics with diffusion length around $50 \mathrm{~nm}$ in Sargent's work [1]. Once diffusion length ( $\left.l_{\text {diff }}\right)$ is known, and we know that the internal electric field $\left(\mathrm{E}_{\text {int }}\right)$ that brings carrier drift is about $2 \sim 3 \times 10^{4} \mathrm{~V} / \mathrm{cm}$, we can also estimate drift length $\left(l_{\text {drift }}\right)$ to be around $200 \mathrm{~nm}\left(1_{\text {drift }}=1_{\text {diff }}^{2} \mathrm{E}_{\text {int }} /(\mathrm{kT} / \mathrm{q})\right.$, where $\mathrm{k}, \mathrm{T}$, and $\mathrm{q}$ are Boltzmann constant, temperature and unit charge.). The estimated drift length is smaller than the onset thickness of full depletion, which shows that the thickness $250 \mathrm{~nm}$ of maximum efficiency (from the fitted graph) is even thinner than the onset thickness of full depletion. This is also related to the drastic reduction of FF with the thickness increase from $200 \mathrm{~nm}$ to $250 \mathrm{~nm}$, while the $\mathrm{FF}$ variation in Sargent's work is more gradual [1]. In their work, diffusion length was estimated to be $100 \mathrm{~nm}$, which corresponds to the drift length of $800 \mathrm{~nm}$. The drift length of amorphous Si solar cells is about ten times the cell thickness [16], which implies that the QDS with atomic passivation still needs three to four times improvement in its drift length to be compatible with state-of-the-art pin type amorphous $\mathrm{Si}$ solar cells.

Material quality of current QDS thin film is still below commercial photovoltaic materials as discussed above. The representative measure of the quality, drift length (or diffusion length) needs to be improved three to four times over that with current technology. Noting that the drift length is proportional to the product of mobility $\mu$ and carrier lifetime $\tau$, and if we want to improve the drift length without a further improvement in QD surface passivation; we can obtain the improvement by controlling QD-to-QD distances [17] and their distributions [18]. For example, the average QD-to-QD distance and their stacking orderness can be reduced by low temperature annealing [19]. More sophisticated processing in coating or post-coating treatment would lead to a higher mobility through more compact stacking of QDs. Maybe in addition, the device structure ought to follow pin type because the carrier collection via drift is more effective in the regime of current QDS quality like amorphous Si solar cells.

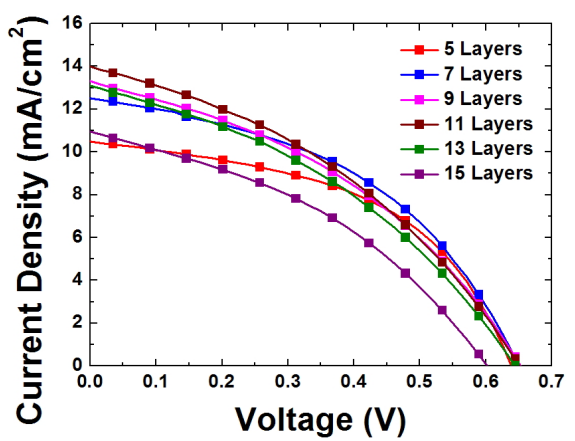

(a)

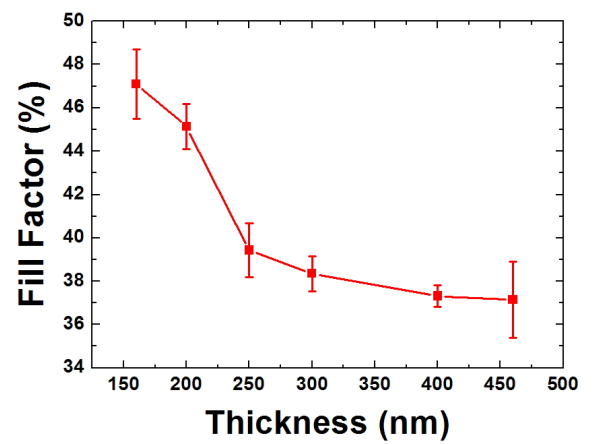

(d)

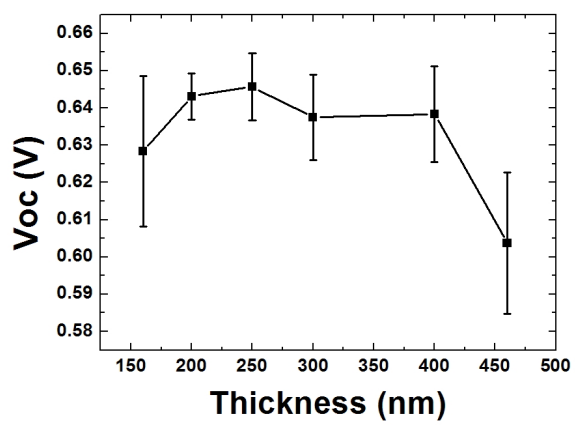

(b)

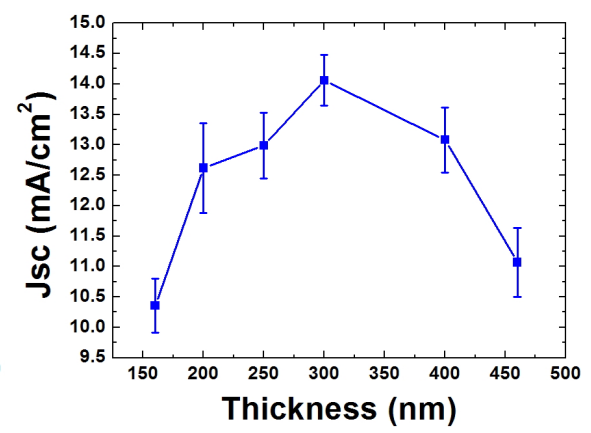

(c)

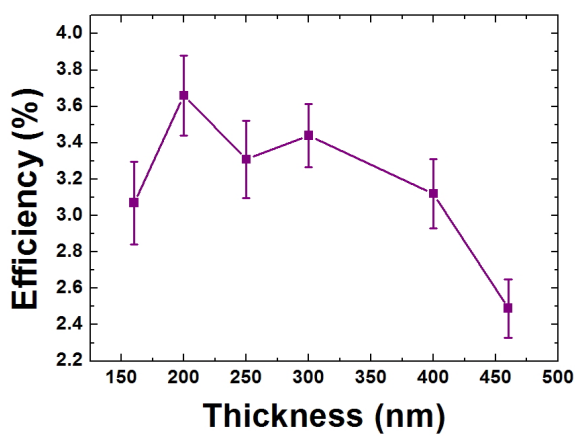

(e)

FIG. 4. (a) Photocurrent density vs. voltage characteristics for a series of solar cells with various numbers of coatings, whose actual thickness ranges from $150 \mathrm{~nm}$ to $450 \mathrm{~nm}$. Thickness dependence of open circuit voltage $\left(\mathrm{V}_{\mathrm{oc}}\right)(\mathrm{b})$, short circuit current $\left(\mathrm{J}_{\mathrm{sc}}\right)(\mathrm{c})$, fill factor (FF) (d), and efficiency (e). 


\section{CONCLUSION}

We have investigated charge transport characteristics of one-step MPA-treated-QDS thin films using the SCLC analysis and the thickness dependent photovoltaic performance assessment. The current level of carrier drift mobility was estimated to be low- $10^{-2} \mathrm{~cm}^{2} / \mathrm{Vs}$ with the diffusion length about 50 $\mathrm{nm}$. The state-of-the-art QDS quality with two-step passivation is known to exhibit two times larger diffusion length, which is still to be improved three to four times in comparison with commercial photovoltaic materials like amorphous Si. A further development in coating method to optimize QD stacking would enable photovoltaic quality QDS compatible with other commercial photovoltaic materials.

\section{ACKNOWLEDGMENT}

This work was supported by the National Research Foundation (NRF) grant funded by the Korean Government (NRF-2013R1 A1A2005765). Y. J and S. J were also supported by Global Frontier R\&D Program by the Center for Multiscale Energy Systems funded by the National Research Foundation under the Ministry of Education, Science, and Technology (20110031566).

\section{REFERENCES}

1. D. Zhitomirsky, O. Voznyy, L. Levina, S. Hoogland, K. W. Kemp, A. H. Ip, S. M. Thon, and E. H. Sargent, "Engineering colloidal quantum dot solids within and beyond the mobility-invariant regime," Nat. Comm. 5, 1-7 (2014).

2. C.-H. M. Chuang, P. R. Brown, V. Bulović, and M. G. Bawendi, "Improved performance and stability in quantum dot solar cells through band alignment engineering," Nat. Mater. 13, 796-801 (2014).

3. I. J. Kramer and E. H. Sargent, "The architecture of colloidal quantum dot solar cells: Materials to devices," Chem. Rev. 114, 863-882 (2014).

4. X. Lan, S. Masala, and E. H. Sargent, "Charge-extraction strategies for colloidal quantum dot photovoltaics," Nat. Mater. 13, 233-240 (2014).

5. A. J. Nozik, "Quantum dot solar cells," Physica E14, 115-120 (2002)

6. D. V. Talapin and C. B. Murray, "PbSe nanocrystal solids for $\mathrm{n}$ - and $\mathrm{p}$-channel thin film field-effect transistors," Science 310, 86-89 (2005).

7. J. T. Stewart, L. A. Padilha, M. M. Qazilbash, J. M. Pietryga, A. G. Midgett, J. M. Luther, M. C. Beard, A. J. Nozik, and V. I. Klimov, "Comparison of carrier multiplication yields in PbS and PbSe nanocrystals: The Role of competing energyloss processes," Nano Lett. 12, 622-628 (2012).

8. O. E. Semonin, J. M. Luther, S. Choi, H. Y. Chen, J. Gao, A. J. Nozik, and M. C. Beard, "Peak external photocurrent quantum efficiency exceeding $100 \%$ via MEG in a quantum dot solar cell," Science 34, 1530-1533 (2011).

9. A. H. Ip, S. M. Thon, S. Hoogland, O. Voznyy, D. Zhitomirsky, R. Debnath, L. Levina, L. R. Rollny, G. H. Carey, A. Fischer, K. W. Kemp, I. J. Kramer, Z. Ning, A. J. Labelle, K. W. Choi, A. Amassian, and E. H. Sargent, "Hybrid passivated colloidal quantum dot solids," Nat. Nanotech. 7, 577-582 (2012).

10. J. Tang, K. W. Kemp, S. Hoogland, K. S. Jeong, H. Liu, L. Levina, M. Furukawa, X. Wang, R. Debnath, D. Cha, K. W. Choi, A. Fischer, A. Amassian, J. B. Asbury, and E. H. Sargent, "Colloidal-quantum-dot photovoltaics using atomic-ligand passivation," Nat. Mater. 10, 765-771 (2011).

11. K. Katsiev, A. H. Ip, A. Fischer, I. Tanabe, X. Zhang, A. R. Kirmani, O. Voznyy, L. R. Rollny, K. W. Choi, S. M. Thon, G. H. Carey, X. Cui, A. Amassian, P. Dowben, E. H. Sargent, and O. M. Bakr, "The complete In-Gap electronic structure of colloidal quantum dot solids and its correlation with electronic transport and photovoltaic performance," Adv. Mater. 26, $937-942$ (2014).

12. P. Stadler, B. R. Sutherland, Y. Ren, Z. Ning, A. Sinchi, S. M. Thon, S. Hoogland, and E. H. Sargent, "Joint mapping of mobility and trap density in colloidal quantum dot solids," ACS Nano 7, 5757-5762 (2013).

13. J. K. Kim, J. H. Song, H. Choi, S. J. Baik, and S. Jeong, "Space charge limited conduction in ultrathin PbS quantum dot solid diodes," J. Appl. Phys. 115, 054302 (2014).

14. H. Choi, J. K. Kim, J. H. Song, Y. Kim, and S. Jeong, "Increased open-circuit voltage in a Schottky device using $\mathrm{PbS}$ quantum dots with extreme confinement," Appl. Phys. Lett. 102, 193902 (2013).

15. H. J. Snaith, A. Abate, J. M. Ball, G. E. Eperon, T. Leijtens, N. K. Noel, S. D. Stranks, J. T.-W. Wang, K. Wojciechowski, and W. Zhang, "Anomalous hysteresis in perovskite solar cells," J. Phys. Chem. Lett. 5, 1511-1515 (2014).

16. S. J. Baik and K. S. Lim, "Nitrogen plasma treatment of fluorine-doped tin oxide for enhancement of photo-carrier collection in amorphous Si solar cells," J. Appl. Phys. 109, 084506 (2011).

17. Y. Liu, M. Gibbs, J. Purthussery, S. Gaik, R. Ihly, H. W. Hillhouse, and M. Law, "Dependence of carrier mobility on nanocrystal size and ligand length in PbSe nanocrystal solids," Nano Lett. 10, 1960-1969 (2010).

18. M. S. Kang, A. Sahu, D. J. Norris, and C. D. Frisbie, "Size-dependent electrical transport in CdSe nanocrystal thin films," Nano Lett. 10, 3727-3732 (2010).

19. S. J. Baik, K. Kim, K. S. Lim, S. M. Jung, Y.-C. Park, D. G. Han, S. Lim, S. Yoo, and S. Jeong, "Low-temperature annealing for highly conductive lead chalcogenide quantum dot solids," J. Phys. Chem. C 115, 607-612 (2011). 